\title{
Biodisponibilidade de ferro em diferentes compostos para leitões desmamados aos 21 dias de idade
}

\author{
Maria Lúcia Cocato ${ }^{1}$, Messias Alves da Trindade Neto ${ }^{2}$, Dirlei Antonio Berto ${ }^{3}$, Maria Inês Ré ${ }^{4}$, \\ Célia Colli 5
}

\footnotetext{
${ }^{1}$ Curso de Pós-Graduação, Departamento de Alimentos e Nutrição Experimental, FCF, USP, Butantã, CEP: 05508-900, São Paulo, SP, Brasil.

2 Departamento de Nutrição e Produção Animal da FMVZ-USP, Campus de Pirassununga, Rua Duque de Caxias Norte, 225, CEP: 13630-000, Pirassununga, SP.

${ }^{3}$ Departamento de Produção e Exploração Animal, FMVZ, UNESP/Botucatu, SP.

${ }^{4}$ Laboratório de Tecnologia de Partículas, IPT, São Paulo,SP, Brasil.

${ }^{5}$ Departamento de Alimentos e Nutrição Experimental, FCF, USP, Butantã, São Paulo, SP, Brasil.
}

RESUMO - Avaliou-se a biodisponibilidade de ferro de diferentes compostos visando sua utilização em dietas para leitões desmamados. Utilizaram-se 44 leitões (7 não-anêmicos e 37 anêmicos) desmamados aos 21 dias de idade (7,3 $\pm 1,8$ kg) e distribuídos em dois grupos: grupo não-anêmico e grupo anêmico. Durante sete dias, os animais do grupo não-anêmico receberam dieta com $\mathrm{FeSO}_{4} \cdot 7 \mathrm{H}_{2} \mathrm{O}$ (sulfato ferroso hepta-hidratado) na dose de $100 \mathrm{mg} / \mathrm{kg}$ e os do grupo anêmico, dieta sem ferro ( $<15 \mathrm{mg} / \mathrm{kg}$ ração). No sétimo dia, depois de determinada a concentração de hemoglobina sanguínea e diagnosticada a anemia, os leitões foram agrupados segundo o produto do peso $(\mathrm{kg}) \times$ hemoglobina $(\mathrm{g} / \mathrm{dL})$ e alojados individualmente, durante 13 dias, em gaiolas para estudos de digestibilidade, onde foram alimentados com seis rações à base de milho e leite em pó: três raçõespadrão com $\mathrm{FeSO}_{4} \cdot 7 \mathrm{H}_{2} \mathrm{O}$ em quantidade equivalente a 80, 150 e $200 \mathrm{mg} \mathrm{Fe} / \mathrm{kg}$ de ração; duas rações experimentais com ferro (150 mg/kg) na forma de $\mathrm{FeSO}_{4}$ microencapsulado com carboximetilcelulose ou de ferro quelado com metionina; e uma controle com ferro (100 mg/kg). O consumo de ração foi medido diariamente. Nos dias 0, 3, 6, 9 e 13 do período de repleção, os animais foram pesados para avaliação do desempenho e o sangue foi coletado para determinação da concentração de hemoglobina. Ao final do ensaio, os animais foram sacrificados e o fígado foi coletado para determinação das concentrações de ferro total, ferro heme e ferro não-heme. As concentrações hepáticas de ferro heme, ferro não-heme e ferro total não diferiram entre os animais, entretanto, os do grupo controle apresentaram excesso de ferro total no fígado, relacionado à dose de ferro injetada nos leitões após o desmame. Em comparação ao $\mathrm{FeSO}_{4} \cdot 7 \mathrm{H}_{2} \mathrm{O}$ não encapsulado, os compostos de ferro microencapsulado com carboximetilcelulose e de ferro quelado com metionina promovem melhor conversão alimentar em leitões desmamados.

Palavras-chave: absorção, hemoglobina, ingrediente alternativo

\section{Bioavailability of iron in different compounds for piglets weaned at 21 days old}

\begin{abstract}
Iron bioavailability from different compounds was evaluated to be used in diets for weaned piglets. Forty four piglets (7 non-anemic and 37 anemic) weaned at 21 days old (7.3 $\pm 1.8 \mathrm{~kg}$ ) were distributed into two groups: non-anemic group and anemic group. During seven days, the animals from non-anemic group were fed diet with ferrous sulfate hepthydrate (FeSO4.7H2O) in the dose of $100 \mathrm{mg} / \mathrm{kg}$ and of the anemic group, diet without iron ( $<15 \mathrm{mg} / \mathrm{kg}$ diet). On the seventh day, after the determination blood hemoglobin concentration and diagnosed with anemia, piglets were grouped according to product of the weight $(\mathrm{kg}) \times$ hemoglobin $(\mathrm{g} / \mathrm{dL})$ and individually housed, for 13 days in cages for digestibility studies, where they were fed with six diets based on corn and powdered milk: three standard diets with FeSO4.7H2O in equivalent amount of 80, 150 and $200 \mathrm{mg} \mathrm{Fe} / \mathrm{kg}$ diet; two experimental diets, one with iron $(150 \mathrm{mg} / \mathrm{kg})$ in form of FeSO4 microencapsulated with carboxymethylcellulose and other chelated with methionine, and a control diet with iron (100 mg/kg). In the days 0 , 3, 6, 9 and 13 of the repletion period, the animals were weighed for performance evaluation and blood was collected to determine the hemoglobin concentration. At the end, the animals were slaughtered and liver was collected for determination of total iron concentrations, Fe-heme and Fe non-heme. Liver concentrations of Fe-heme, Fe non-heme and Fe-total did not differ among animals, however, the control group showed excess of Fe-total in the liver, related to iron injected dose in the piglets after weaning. Compared to FeSO4.7H2O not encapsulated, iron compounds microencapsulated with carboxymethylcellulose and iron chelate with methionine promote better feed conversion in weaned piglets.
\end{abstract}

Key Words: absorption, alternative ingredient, hemoglobin 


\section{Introdução}

A deficiência de ferro do suíno está associada às condições de confinamento e, nas primeiras semanas de vida, é comum o leitão ter anemia (hipocrômica microcítica) (Kegley et al., 2002). Entre as causas dessa anemia, destacam-se: o baixo estoque de ferro do suíno ao nascimento (aproximadamente $50 \mathrm{mg}$ ), os reduzidos níveis de ferro no leite da porca e a elevada taxa de crescimento do animal (Underwood \& Suttle, 1999). Após o nascimento, cerca de $80 \%$ do micromineral se associa à hemoglobina. O ferro liga-se a proteínas como os complexos heme (hemoglobina e mioglobina) e não-heme (ferritina e hemossiderina). As proteínas contendo ferro são essenciais no transporte de oxigênio, na produção de ATP, na síntese de ácidos nucléicos e outras proteínas (Crosby, 1979).

Ao desmame, grande parte dos leitões tem anemia, provavelmente em razão das falhas na aplicação (refluxo do medicamento) ou dos erros na dosagem do ferro suplementar. A suplementação oral, nesse caso, torna-se alternativa para recompor o teor de ferro orgânico e favorer o crescimento, em uma fase de alta síntese metabólica. O NRC (1998) preconiza a ingestão diária de 7 a 16 mg de ferro, que é absorvido de acordo com as necessidades metabólicas, porém sua biodisponibilidade depende da solubilidade do composto no trato digestório (Underwood \& Suttle, 1999).

O ferro dietético existe nas duas formas: ferro-heme, derivado da hemoglobina e da mioglobina (elevada biodisponibilidade); e ferro não-heme, presente principalmente nos vegetais. O ferro não-heme ocorre na valência férrica $\left(\mathrm{Fe}^{3+}\right)$, tem baixa biodisponibilidade e deve ser primeiro reduzido à valência ferrosa $\left(\mathrm{Fe}^{2+}\right)$, que é solúvel no pH do lúmen intestinal, portanto, mais biodisponível.

A fonte de ferro mais usada nas rações é o $\mathrm{FeSO}_{4} \cdot{ }^{7} \mathrm{H}_{2} \mathrm{O}$. Apesar da alta biodisponibilidade, forma grandes cristais cujas propriedades reológicas não permitem boa distribuição nas rações. Devido à elevada reatividade, o composto possui grande potencial pró-oxidante e reage com outros componentes da ração, produzindo características organolépticas indesejáveis ou se torna menos disponível para absorção.

Não há na literatura estudos que busquem fontes alternativas de Fe para elevada biodisponibilidade e distribuição na ração de forma homogênea, garantindo adequada ingestão diária do mineral pelo suíno.

Neste estudo, avaliou-se a biodisponibilidade de diferentes compostos de ferro para leitões desmamados aos 21 dias de idade.

\section{Material e Métodos}

O experimento foi realizado no setor de suínos do Instituto de Zootecnia, em Nova Odessa, São Paulo. Foram utilizados 44 leitões (7 não-anêmicos e 37 anêmicos) provenientes de granja comercial, mestiços, machos castrados e fêmeas, desmamados aos 21 dias de idade e pesando $7,3 \pm 1,8$ kg. Para indução da anemia, os leitões dos grupos experimentais receberam, ao nascer, injeção de $60 \mathrm{mg} \mathrm{Fe}$ dextran/kg peso corporal. Os leitões do grupo controle receberam a dose de ferro normalmente aplicada de $200 \mathrm{mg}$ de Fe-dextran/kg. Durante sete dias, os leitões do grupo controle (não-anêmicos) receberam dieta com $100 \mathrm{mg} \mathrm{Fe} / \mathrm{kg}$ na forma $\mathrm{FeSO}_{4} \cdot 7 \mathrm{H}_{2} \mathrm{O}$ e os demais, dieta sem ferro $(<15 \mathrm{mgFe} /$ kg ração). Na formulação da dieta (Tabela 1 ), foram adotadas as orientações do Nutritional Research Center (1998).

No sétimo dia, os animais foram pesados e o sangue foi coletado para determinação da concentração de hemoglobinas. Confirmada a anemia, foram alojados individualmente por 13 dias em gaiolas para estudos de digestibilidade, em ambiente com temperatura controlada, em torno de $25^{\circ} \mathrm{C}$.

Tabela 1 - Composição centesimal das dietas-bases de depleção e repleção

\begin{tabular}{|c|c|}
\hline Ingrediente & $(\%)$ \\
\hline Milho moído ${ }^{1}$ & 58,11 \\
\hline Leite em pó desnatado & 40,10 \\
\hline Cloreto de colina & 0,08 \\
\hline Fosfato bicálcico ${ }^{1}$ & 0,49 \\
\hline Carbonato de cálcio & 0,42 \\
\hline Sal & 0,20 \\
\hline L-triptofano & 0,02 \\
\hline L-lisina $\mathrm{HCl}$ & 0,07 \\
\hline Suplemento vitamínico & 0,50 \\
\hline \multicolumn{2}{|l|}{ Mineral (g/100 kg ração) } \\
\hline $\mathrm{ZnO}$ & 13,7 \\
\hline $\mathrm{CuSO}_{4} \cdot 5 \mathrm{H}_{2} \mathrm{O}$ & 2,4 \\
\hline $\mathrm{MnSO}_{4} \cdot \mathrm{H}_{2} \mathrm{O}$ & 1,2 \\
\hline \multicolumn{2}{|l|}{ Composição calculada } \\
\hline Energia metabolizável (kcal/kg) & 3382 \\
\hline Proteína bruta (\%) & 18,13 \\
\hline Cálcio (\%) & 0,80 \\
\hline Fósforo total (\%) & 0,65 \\
\hline Fósforo disponível (\%) & 0,51 \\
\hline Lisina digestível (\%) & 1,19 \\
\hline Metionina+cistina digestível (\%) & 0,73 \\
\hline Triptofano digestível (\%) & 0,22 \\
\hline Treonina digestível (\%) & 0,76 \\
\hline
\end{tabular}


O delineamento foi em blocos ao acaso, formados segundo o produto peso $(\mathrm{kg}) \times$ hemoglobina $(\mathrm{g} / \mathrm{dL})$. Avaliaram-se seis rações, à base de milho e leite em pó: três com $\mathrm{FeSO}_{4} \cdot 7 \mathrm{H}_{2} \mathrm{O}$ para suprimento de 80,150 e $200 \mathrm{mg}$ Fe/kg de ração para formação da curva de absorção × concentração de ferro; duas para suprimento de 150 mg de Fe/kg de ração, uma na forma de $\mathrm{FeSO}_{4}$ microencapsulado com carboximetilcelulose sódica ( $\mathrm{NaCMC}$ ) e outra com ferro quelado com metionina; e uma ração controle.

A microencapsulação é definida como tecnologia de acondicionamento em miniatura de materiais sólidos, líquidos ou gasosos em cápsulas seladas que podem liberar seu conteúdo a uma razão controlada, sob condições específicas (Sparks, 1981; Dziezak, 1988). Entre outras características, esse processo pode reduzir a reatividade de compostos adicionados aos alimentos (acidulantes, ácido ascórbico, vitaminas e minerais) e diluir o material quando é usado em reduzida quantidade e se deseja a dispersão uniforme do elemento no alimento onde foi adicionado (Shahidi \& Han, 1993).

O consumo de ração foi medido diariamente. O cálculo do desempenho e a coleta de sangue dos animais para determinação da hemoglobina foram feitos nos dias 0 , 3, 6, 9 e 13 do período de repleção. A avaliação da biodisponibilidade de ferro foi realizada pelo teste de repleção de hemoglobina em leitões anêmicos, proposto por South et al. (2000). Nesse teste, é determinada a relação entre a variação do pool de ferro do sangue e o ferro ingerido pelos animais para cada fonte de ferro testada. Assumindo-se que o volume de sangue corresponde a $6 \%$ do peso do animal e que a molécula de hemoglobina tem $0,335 \%$ de ferro, o pool de ferro foi calculado como:

Pool de Fe $(\mathrm{mg})=\mathrm{p}(\mathrm{kg}) \times \mathrm{Hb}(\mathrm{g} / \mathrm{L}) \times 0,06 \times 3,35$ em que: $\mathrm{p}=$ peso do leitão $(\mathrm{kg}) ; \mathrm{Hb}=$ hemoglobina $(\mathrm{g} / \mathrm{L})$

O sangue coletado foi transferido para tubos contendo $\mathrm{K}_{3}$ EDTA como solução anticoagulante, mantidos sob refrigeração, sem agitação, até a determinação da concentração de hemoglobina, feita no Laboratório Zoológica de Campinas (SP), em duplicata, utilizando-se padrão de hemoglobina de $10 \mathrm{~g} / \mathrm{dL}$ (Labtest ${ }^{\circledR}$ ).

O método de repleção de hemoglobina em ratos anêmicos, proposto por Fritz et al. (1975) para determinação da biodisponibilidade de ferro, adequa-se ao estudo com leitões (South et al., 2000). No método, a anemia dos animais é induzida por determinado período de depleção com uma dieta pobre em ferro. Paralelamente, até o final do experimento, mantém-se um grupo controle de animais não anêmicos. Em seguida, são fornecidas dietas-teste contendo ferro das fontes em estudo e grupos padrão com dietas com FeSO4.7H2O (padrão de referência) em diferentes propor- ções. Esse período é denominado período de repleção. A repleção de hemoglobina dos animais alimentados com a dieta-teste é avaliada pelo aumento do pool de ferro no sangue e comparada às dos animais do grupo padrão. Os resultados são expressos como Valor Biológico Relativo, ou biodisponibilidade relativa. Como a biodisponibilidade é a proporção absorvida do nutriente pelo organismo, proveniente do alimento ou dieta usada (Benito \& Miller, 1998), o aumento do pool de ferro torna-se a medida da biodisponibilidade do elemento na dieta. Além da fonte, a biodisponibilidade varia com a idade e a espécie animal, o estado nutricional em ferro e fatores dietéticos promotores, como ácido ascórbico, e inibidores, como taninos, fitatos, fosfatos e oxalato (Hallberg, 2001).

Ao final do ensaio, os animais foram sacrificados para retirada do fígado, que foi pesado e acondicionado a $-20^{\circ} \mathrm{C}$ até o momento da análise.

$\mathrm{OFeSO}_{4} \cdot 7 \mathrm{H}_{2} \mathrm{Omicroencapsulado} \mathrm{com} \mathrm{carboximetilcelulose}$ sódica (NaCMC) foi preparado pelo Laboratório de Caracterização de Partículas do Agrupamento de Processos Químicos, no Instituto de Pesquisas Tecnológicas da Universidade de São Paulo (IPT), pelo método spray drying. O ferro quelado com metionina foi fornecido pela empresa Fermavi Eletroquímica Ltda.

Análises estatísticas dos dados foram realizadas utilizando-se o pacote computacional SAS (1996).

\section{Resultados e Discussão}

Na avaliação das concentrações de ferro nas rações de repleção, constatou-se que o composto microencapsulado conferiu maior uniformidade de distribuição do sal de ferro nas dietas ( $\mathrm{CV}=4 \%$ ) (Tabela 2). Em estudo preliminar, Cocato et al. (2007) constataram que a microencapsulação do FeSO4 com derivado de polissacarídeos (Ferlim ${ }^{\circledR}$ ) proporcionou excelente distribuição do mineral na ração.

Não houve diferenças $(\mathrm{P}>0,05)$ entre os grupos para a concentração final de hemoglobina (Tabela 3), mas a esperada diminuição na porcentagem de absorção de ferro ocorreu com o aumento da concentração desse mineral na dieta, o que indica fino controle na absorção do mineral. A absorção de ferro das rações-padrão em relação à ingestão de ferro ajustou-se à equação:

$$
\hat{U}=48,77 .\left(e^{-0,0013 X}\right)\left(r^{2}=0,9997\right)
$$

em que $X$ é o total de ferro ingerido no período de repleção e Û, a porcentagem de absorção. Assim, calculou-se a porcentagem de absorção do grupo-padrão correspondente a cada nível de ingestão individual dos animais dos grupos experimentais. A absorção de ferro das rações-padrão foi tomada como $100 \%$ e possibilitou calcular a absorção relativa. 
Tabela 2 - Concentração de ferro nas rações de repleção

\begin{tabular}{|c|c|c|c|c|}
\hline Grupo & $\mathrm{n}$ & Fonte de ferro & mg Fe/kg dieta & CV (\%) \\
\hline PFe $80^{1}$ & 5 & $\mathrm{FeSO}_{4} \cdot 7 \mathrm{H}_{2} \mathrm{O}$ & $77,5(24,3) a$ & 31 \\
\hline PFe $150^{1}$ & 5 & $\mathrm{FeSO}_{4} \cdot 7 \mathrm{H}_{2} \mathrm{O}$ & $141,4(38,3) b$ & 27 \\
\hline $\mathrm{PFe} 200^{1}$ & 5 & $\mathrm{FeSO}_{4} \cdot 7 \mathrm{H}_{2} \mathrm{O}$ & $214,9(67,6) b, c$ & 31 \\
\hline Fe quelado & 5 & Fe quelado com metionina & $156,7(64,1) a, b, c, d$ & 41 \\
\hline Controle & 5 & $\mathrm{FeSO}_{4} \cdot 7 \mathrm{H}_{2} \mathrm{O}$ & $97,5(14,1) a, c, d$ & 15 \\
\hline
\end{tabular}

Média (DP) e coeficiente de variação (\%).

Médias seguidas de mesma letra na coluna não diferem entre si pelo teste de Tukey (5\%)

${ }^{1}$ Grupos-padrão de ferro (rações com $\mathrm{FeSO}_{4} \cdot 7 \mathrm{H}_{2} \mathrm{O}$ em diferentes concentrações).

${ }^{2}$ Carboximetilcelulose de sódio.

Tabela 3 - Recuperação de hemoglobina em leitões anêmicos ${ }^{1}$

\begin{tabular}{|c|c|c|c|c|}
\hline Parâmetro & Dia & Padrão Fe 80 & Padrão 150 & Padrão 200 \\
\hline \multirow[t]{3}{*}{ Peso (kg) } & 0 & $7,8 \quad(1,3) a$ & $8,1 \quad(1,2) a$ & $7,7 \quad(0,9) a$ \\
\hline & 6 & $9,9(1,9) b$ & $10,0(1,6) \mathrm{b}$ & $9,1(1,3) b$ \\
\hline & 13 & $11,8(2,1) \mathrm{c}$ & $11,6(1,7) \mathrm{c}$ & $11,3(1,3) \mathrm{c}$ \\
\hline \multirow[t]{3}{*}{ Hemoglobina (g/L) } & 0 & $63,9(6,2) a$ & $60,3(10,7) \mathrm{a}$ & $64,4(7,5) \mathrm{a}$ \\
\hline & 6 & $83,7(9,7) b$ & $81,1 \quad(12,2) b$ & $86,9(10,3) b$ \\
\hline & 13 & $94,8 \quad(9,1) \mathrm{c}$ & $100,9(10,0) \mathrm{c}$ & $97,2(8,7) \mathrm{c}$ \\
\hline \multirow[t]{3}{*}{ POOL-Fe (mg) } & 0 & $99,0 \quad(14,3) \mathrm{a}$ & $99,1 \quad(23,3) a$ & $99,0 \quad(10,7) \mathrm{a}$ \\
\hline & 6 & $165,5(27,8) b$ & $165,2(44,3) \mathrm{b}$ & $159,1(28,4) b$ \\
\hline & 13 & $224,3(44,8) \mathrm{c}$ & $236,9(49,7) \mathrm{c}$ & $221,2(42,2) c$ \\
\hline Fe ingerido (mg) & 13 & $498,0 \quad(124,6) a$ & $861,8(159,4) b$ & $1220,2(203,4) c$ \\
\hline Absorção (\%) & & $25,4(4,3) a$ & $16,0(3,1) b$ & $9,9(2,2) \mathrm{c}$ \\
\hline
\end{tabular}

Grupos - padrão de $\mathrm{FeSO}_{4} \cdot 7 \mathrm{H}_{2} \mathrm{O}$. Média (DP).

Médias seguidas de mesma letra na linha não diferem entre si pelo teste de Tukey $(5 \%)$

${ }^{1}$ Leitões não-anêmicos alimentados durante 20 dias com rações fortificadas com $\mathrm{FeSO}_{4} \cdot 7 \mathrm{H}_{2} \mathrm{O}$

Utilizando essa equação, garantiu-se que as diferenças na absorção fossem relacionadas somente a diferenças de biodisponibilidade das fontes de ferro, e não a diferenças de consumo do mineral (Pinto et al., 1997).

Excetuando a hemoglobina inicial $(\mathrm{P}<0,05)$ e o ferro ingerido $(\mathrm{P}<0,001)$ do grupo controle, não houve diferença $(\mathrm{P}>0,05)$ entre os grupos estudados em todos os períodos da repleção (Tabela 4).

Acredita-se que a utilização de ferro na forma de quelatos proteja o mineral da reação com diversos constituintes da dieta, aumentando, portanto, a sua biodisponibilidade (Fox et al., 1998; Pineda \& Ashmead, 2001). Segundo Ashmead \& Jeppsen (1993), a maior biodisponibilidade do ferro glicina quelato ocorreria por este composto ser absorvido intacto pelas células epiteliais do intestino, provavelmente via transporte ativo. Neste estudo, a absorção do ferro quelado com metionina foi similar à do FeSO4.7H2O, o que está de acordo com as informações desses autores.

Em leitões não anêmicos, Anderson et al. (1974) avaliaram a biodisponibilidade de várias fontes de ferro e obtiveram para o sulfato ferroso absorção de $30 \%$ do ferro. Howard et al. (1993), trabalhando com o mesmo modelo animal, obtiveram absorção de 19,8\% para o sulfato ferroso. Essas diferenças se devem provavelmente a diferenças nas formulações das rações.

Não houve diferenças $(\mathrm{P}>0,05)$ na ingestão diária de ração e no ganho de peso entre os grupos (Tabela 5). Houve, porém, indicação não-significativa de menor ingestão de ração pelos animais do grupo NaCMC (408 \pm $96 \mathrm{~g} / \mathrm{dia}$ ), o que não refletiu no ganho de peso. Em comparação aos leitões dos grupos-padrão PFe80, PFe150 e $\mathrm{PFe} 200$ que receberam dieta com $\mathrm{FeSO}_{4} .7 \mathrm{H}_{2} \mathrm{O}$ e ingeriram maior quantidade de ração por dia $(494 \pm 124,469 \pm 87$ e $437 \pm 73 \mathrm{~g}$ /dia, respectivamente), os resultados do grupo NaCMC sugeriram $(\mathrm{P}>0,05)$ maior ganho de peso diário $(317 \pm 69 \mathrm{~g} /$ dia vs $309 \pm 77,267 \pm 75$ e $274 \pm 45 \mathrm{~g} /$ dia, respectivamente).

Os leitões que consumiram dietas fortificadas com ferro microencapsulado com NaCMC e ferro quelado mostraram maior $(\mathrm{P}<0,05)$ eficácia $(1,3 \pm 0,1)$ no uso dos nutrientes dietéticos em comparação aos dos grupos-padrão, PFe80 e PFe200 $(1,6 \pm 0,1)$. 
Tabela 4 - Recuperação de hemoglobina em leitões anêmicos (Média, desvio-padrão)

\begin{tabular}{|c|c|c|c|c|}
\hline & & Carboximetilcelulose $\mathrm{Na}$ & Fe quelado & Controle \\
\hline \multirow[t]{3}{*}{ Peso (kg) } & 0 & $6,8 \quad(0,6) a$ & $6,5(1,1) a$ & $7,1 \quad(1,4) a$ \\
\hline & 6 & $8,3(0,8) b$ & $8,5(1,8) b$ & $9,2(1,4) b$ \\
\hline & 13 & $10,9(1,2) \mathrm{c}$ & $11,2(2,9) \mathrm{c}$ & $11,6(1,8) \mathrm{c}$ \\
\hline \multirow[t]{3}{*}{ Hemoglobina (g/L) } & 0 & $68,7(10,5) a$ & $71,2(12,2) a, b$ & $88,6(8,6) b$ \\
\hline & 6 & $79,7(9,7) b$ & $84,6(11,4) b$ & $100,4(13,4) b$ \\
\hline & 13 & $96,7 \quad(8,2) c$ & $96,4 \quad(14,4) \mathrm{c}$ & $110,4(14,1) \mathrm{c}$ \\
\hline \multirow[t]{3}{*}{ POOL Fe (mg) } & 0 & $93,2(11,7) a$ & $91,4(16,9) a$ & $127,8(31,3) b$ \\
\hline & 6 & $133,0(23,5) b$ & $145,6(42,2) b$ & $185,1(30,1) b$ \\
\hline & 13 & $213,4(36,1) \mathrm{c}$ & $219,6(73,9) \mathrm{c}$ & $256,3(46,5) c$ \\
\hline Fe ingerido (mg) & 13 & $823,0 \quad(194,7) \mathrm{a}$ & $934,0 \quad(388,0) a$ & $521,7 \quad(96,0) b$ \\
\hline Absorção (\%) & & $14,7 \quad(2,9) a$ & $13,3 \quad(2,9) a$ & \\
\hline Absorção relativa (\%) & & $89,5(25,9) a$ & $105,9(60,5) a$ & \\
\hline
\end{tabular}

Médias seguidas de mesma letra na linha não diferem entre si pelo teste Tukey (5\%).

${ }^{1}$ Leitões não-anêmicos alimentados durante 20 dias com rações fortificadas com $\mathrm{FeSO}_{4} \cdot 7 \mathrm{H}_{2} \mathrm{O}$.

Tabela 5 - Desempenho dos leitões no período de repleção

\begin{tabular}{lcccc}
\hline Grupo & $\mathrm{N}$ & Consumo ração (g/dia) & Ganho em peso (g/dia) & Conversão alimentar \\
\hline PFe80 & 7 & $494,1(123,7) \mathrm{a}$ & $309(77) \mathrm{b}$ & $1,6(0,1) \mathrm{c}$ \\
PFe150 & 7 & $468,7(86,7) \mathrm{a}$ & $267(75) \mathrm{b}$ & $1,8(0,4) \mathrm{c}$ \\
PFe200 & 7 & $436,7(72,8) \mathrm{a}$ & $274(45) \mathrm{b}$ & $1,6(0,1) \mathrm{c}$ \\
NaCMC & 8 & $407,9(96,5) \mathrm{a}$ & $317(69) \mathrm{b}$ & $1,3(0,1) \mathrm{d}$ \\
Fe quelado & 8 & $458,6(190,5) \mathrm{a}$ & $363(156) \mathrm{b}$ & $1,3(0,3) \mathrm{d}$ \\
Controle & 7 & $410,1(75,5) \mathrm{a}$ & $341(51) \mathrm{b}$ & $1,3(0,2) \mathrm{d}$ \\
\hline
\end{tabular}

Médias seguidas de mesma letra na coluna não diferem entre si pelo teste de Tukey (5\%).

Média (D.P.)

Esses resultados sugerem que, independentemente da concentração de ferro das dietas, houve similaridade entre os grupos de leitões quanto à ingestão de ferro e quanto ao ganho de peso. Estes dados correspondem aos obtidos por outros autores, quando observaram que a redução de ferro nas rações não alterou o ganho de peso dos animais em fase de crescimento alimentados com a mesma fonte de ferro em diferentes concentrações (Amine et al., 1972). Essas observações foram destacadas em estudos com leitões após o desmame (Yu et al., 2000; Rincker et al., 2004).

Em leitões anêmicos, não foram encontrados na literatura consultada trabalhos relacionando os índices de conversão ou eficiência alimentar com dietas com diferentes concentrações de ferro. Furugouri (1972), trabalhando com leitões não-anêmicos alimentados durante 30 ou 60 dias com dietas contendo concentrações de Fe de 200 a $7.000 \mathrm{mg} / \mathrm{kg}$, na forma de sulfato ferroso, não encontrou diferença na ingestão diária de ração entre os grupos nem no ganho de peso diário. Os índices de conversão ou eficiência alimentar, no entanto, não foram considerados.
Com exceção do grupo controle, que apresentou maior $(\mathrm{P}<0,05)$ concentração de ferro total e ferro não-heme no fígado, não houve diferença $(\mathrm{P}>0,05)$ entre os grupos estudados quanto ao peso do fígado, as concentrações de ferro total, ferro heme e ferro não-heme (Tabela 6). Houve, todavia, indicação de maior concentração de ferro total e de ferro não-heme nos grupos PFe150 e PFe200, o que confirma a capacidade do sulfato ferroso como fonte de ferro com alta biodisponibilidade. Por outro lado, a elevada concentração de ferro total no fígado dos leitões do grupo controle indica excesso na dose de ferro aplicada aos animais, o que pode ocasionar sobrecarga hepática de ferro e danos oxidativos no tecido.

Trabalhando com leitões anêmicos, recém-desmamados, Yu et al. (2000) encontraram nos grupos PFe resultados de ferro total, ferro heme e ferro não-heme semelhantes aos dos leitões que receberam dietas com sulfato ferroso.

No entanto, o menor valor de ferro total no fígado dos animais do grupo $\mathrm{PFe} 80$ pode estar relacionado à menor concentração de ferro na ração desse grupo (77,5 mg Fe/kg 
Tabela 6 - Parâmetros corporais de ferro dos leitões no 13ํ dia de recuperação (média, desvio-padrão)

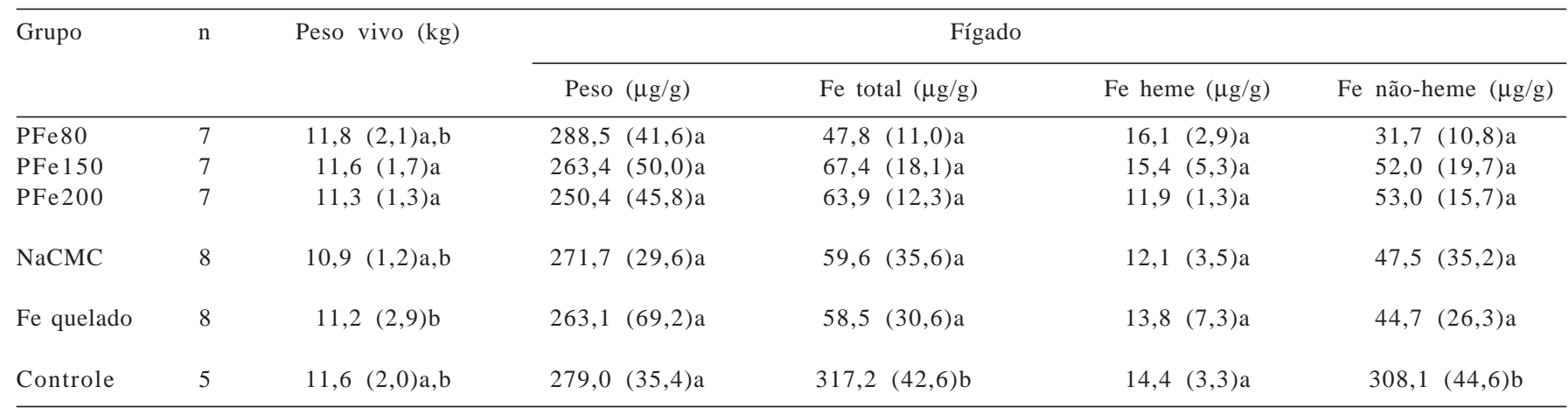

Médias seguidas de mesma letra na coluna não diferem entre si pelo teste Tukey (5\%)

ração) sugerindo-se que grande parte do ferro dietético foi utilizada na síntese de hemoglobina, não havendo quase reserva hepática.

Neste trabalho, além da biodisponibilidade de ferro das diferentes fontes, os resultados comprovaram que pode haver excesso de ferro no fígado de leitões quando submetidos à injeção de ferro (200 mg/kg) seguida de consumo dietético de ferro (em torno de $100 \mathrm{mg} / \mathrm{kg}$ dieta) após o desmame. Comprovaram também que é possível reduzir a quantidade de ferro injetado se o FeSO4 for acrescentado à ração, na forma microencapsulado com carboximetilcelulose, na concentração de 150 mg Fe/kg.

\section{Conclusões}

As fontes avaliadas se equivalem quanto à biodisponibilidade de ferro. O FeSO4.7H2O protegido pela microencapsulação com carboximetilcelulose propicia melhor a distribuição do ferro nas rações e melhora a conversão alimentar em comparação ao não encapsulado. Embora não tenha sido o objetivo principal do trabalho, os resultados mostraram que, quando o leitão é desmamado anêmico e uma das duas fontes de ferro testadas neste trabalho é utilizada na ração, é recomendável reduzir a quantidade de ferro suplementar injetado nos leitões após o desmame.

\section{Agradecimentos}

À FERMAVI e ao Instituto de Zootecnia da APTASAA-SP.

\section{Literatura Citada}

AMINE, E.K.; RAYMOND, N.; HEGSTED, D.M. Biological estimation of available iron using chicks or rats. Journal of Agriculture Food Chemistry, v.20, n.2, p.246-251, 1972.
ANDERSON, B.K.; EASTER, R.A. [1999]. A review of iron nutrition in pigs. <http://www.livestochtraill.uiuc.edu/poknet/paperDisplay. cfm?ContentID=70> Acesso em: 18/2/2008.

ANDERSON, T.A.; FILER, L.J.; FOMON, S.J. et al. Bioavailability of different sources of dietary iron fed to Pitman-Moore miniature pigs. Journal of Nutrition., v.104, n.5, p.619-627, 1974.

ASHMEAD, H.D.; JEPPSEN, R.B. Enhanced tissue metabolism of minerals chelated to amino acids. In: SCHLEMMER, U. (Ed.) Bioavailability '93, nutritional, chemical and food processing implications of nutrient availability. Part 2. Ettlingen, Germany: Bundes-forschungsanstalt fur Ernahrung, 1993. p.63-67.

BENITO, P.; MILLER, D. Iron absorption and bioavailability: an update review. Nutrition Research, v.18, n.3, p.581-603, 1998.

COCATO, M.L.; RÉ, M.I.; TRINDADE NETO, M.A. et al. Avaliação por métodos in vitro e in vivo da biodisponibilidade de sulfato ferroso microencapsulado. Revista de Nutrição, v.20, n.3, p.239-247, 2007.

CONRAD, M.E.; UMBREIT, J.N. Pathways of iron absorption. Blood Cells, Molecules, and Diseases, v.29, n.3, p.336-355, 2002.

CONRAD, M.E.; UMBREIT, J.N.; MOORE, E.G. Iron absorption and transport. American Journal of Medicine Science, v.318, n.4, p.213-229, 1999.

CROSBY, W.H. Iron deficiency anaemia in a nutritionally complex situation. American Journal of Clinical Nutrition, v.32, n.4, p.715-716, 1979.

DZIEZAK, J.D. Microencapsulation and encapsulated ingredients. Food Technology, v.42, n.3-4, p.137-151, 1988.

FOX, T.E.; FAIRWEATHER-TAIT, S.J. Bioavailability of iron glycine as a fortificant in infant food. American Journal of Clinical Nutrition, v.67, n.4, p.664-668, 1998.

FRITZ, J.C.; PLA, G.W.; HARRISON, B.N. et al. Estimation of the bioavailability of iron. Journal of the Association of Official Analytical Chemists, v.58, n.5, p.902-905, 1975.

FURUGOURI, K. Effect of elevated dietary levels of iron on iron store in liver, some blood constituents and phosphorus deficiency in young swine. Journal of Animal Science, v.34, n.4, p.573-577, 1972.

HALLBERG, L. Perspectives of nutritional iron deficiency. Annual Review of Nutrition, v.21, n.1, p.1-21, 2001.

KEGLEY, E.B.; SPEARS, J.W.; FLOWERS, W.L. et al. Iron methionine as a source of iron for the neonatal pig. Nutrition Research, v.22, n.10, p. 1209-1217, 2002.

NATIONAL RESEARCH COUNCIL - NRC. Nutrient requirements of swine. 10.ed. Washington, D.C.: National Academy of Science, 1998. 189p.

PINEDA, O.; ASHMEAD, H.D. Effectiveness of treatment of irondeficiency anemia in infants and young children with ferrous bis-glycinate chelate. Nutrition, v.17, n.5, p.381-384, 2001. 
PINTO, T.A.; COLLI, C.; AREAS, J.A.G. Effect of processing on iron bioavailability of extruded bovine lung. Food Chemistry, v.60, n.4, p.459-463, 1997.

RINCKER, M.J.; HILL, G.M.; LINK, J.E. et al. Effects of dietary iron supplementation on growth performance, hematological status, and whole-body mineral concentrations of nursery pigs. Journal of Animal Science, v.82, n.11, p.3189-3197, 2004.

STATISTICAL ANALYSIS SYSTEM - SAS. User's guide: statistics. Version 6.12. Cary: SAS Institute, 1996. 956p.

SHAHIDI, F.; HAN, X.Q. Encapsulation of food ingredients. Critical Reviews in Food Science Nutrition, v.33, n.6, p.501-547, 1993.
SOUTH, P.K.; LEI, X.; MILLER, D.D. Meat enhances nonheme iron absorption in pigs. Nutrition Research, v.20, n.12, p.1749-1759, 2000.

SPARKS, R.E. Microencapsulation. In: KIRK-OTHMER, E. (Ed.) Encyclopedia of chemical technology. 3.ed. New York: Wiley, 1981. p.470-493.

UNDERWOOD, E.J.; SUTTLE N.F. Mineral nutrition of livestock. 3.ed. Edinburgh: CABI Publishing, 1999. 624p.

YU, B.; HUANG, W.J.; CHIOU, P.W.S. Bioavailability of iron from amino acid complex in wealing pigs. Animal Feed Science and Technology, v.86, n.1-2, p.39-52, 2000. 